\title{
ПОЛУЧЕНИЕ ОТЕЧЕСТВЕННОГО ПРЕПАРАТА РЕКОМБИНАНТНОГО ТИРЕОТРОПНОГО ГОРМОНА ЧЕЛОВЕКА ДЛЯ ИСПОЛЬЗОВАНИЯ В КЛИНИЧЕСКОЙ ПРАКТИКЕ
}

\author{
${ }^{1,2}$ Смирнов И.В., ${ }^{1}$ Бобик Т.В., ${ }^{1}$ Костин Н.Н., ${ }^{2}$ Трошина Е.А., ${ }^{1}$ Габибов А.Г., ${ }^{2}$ Дедов И.И.
}

'Федеральное государственное бюджетное учреждение науки Институт биоорганической химии им. академиков М.М. Шемякина и Ю.А. Овчинникова Российской академии наук (ИБХ РАН)

2 Федеральное государственное бюджетное учреждение «Начиональный медицинский исследовательский иентр эндокринологии» Министерства здравоохранения Российской Федерации (ФГБУ «НМИЦ эндокринологии» Минздрава России)

Основные функции тиреотропного гормона (ТТГ) гипофиза - поддерживать биосинтез и секрецию тиреоидных гормонов L-тироксина (T4) и L-3,5,3'-трийодтиронина (Т3). Использование ТTГ показано при подготовке к радиойоддиагностике и радиойодтерапии пациентов с раком щитовидной железы. Экзогенная стимуляция рекомбинантным ТТГ человека (рчТТГ) не вызывает побочных эффектов при этом процессе, однако стоимость такой процедуры весьма высока. На рынке наиболее известным рчТТГ является зарубежный препарат Тироджин (Thyrogen ${ }^{\text {TM}}$, Sanofi Genzyme). Целью нашей работы является создание отечественного аналога рчТТГ для последующей организации импортозамещающего производства ГЛС, доклинических и клинических исследований, получение регистрации в РФ и применение в клинической практике. В ходе текущего этапа работ была получена генетическая конструкция PFUSE X-29 TSH, содержащая гены $\alpha$ - и $\beta$-субъединиц под контролем сильного гибридного промотера и уникальную комбинацию элементов. Была разработана стратегия селекции линий клеток-продуцентов, позволяющая добиться продукции рчТТГ в количестве 41.5 мг/л в качалочных колбах. Ранее полученные данные дают уверенность, что после получения индивидуальных клонов-продуцентов из селектированной на данном этапе линии, будет повышен уровень продукции более чем в 10 раз. 